\title{
Colorectal Services in Covid-19 Times: Minimally Invasive Surgery and Enhanced Recovery, the Need of the Hour
}

\author{
Vivek Sukumar ${ }^{1} \cdot$ Diwakar Pandey $^{1} \cdot$ Barath Raj Kumar $^{1} \cdot$ Swapnil Patel ${ }^{1} \cdot$ Tushar Pawar $^{1} \cdot$ Jitender Rohila $^{1}$. \\ Ashwin DeSouza ${ }^{1} \cdot$ Avanish Saklani $^{1}$
}

Received: 19 June 2020 / Accepted: 21 September 2020 / Published online: 14 October 2020

(C) Indian Association of Surgical Oncology 2020

\begin{abstract}
The health services across the world have been deeply impacted by the ongoing COVID-19 pandemic which has resulted in diversion of resources to testing, isolating and treating COVID-19 patients. This meant cutting down resources and manpower away of various healthcare facilities and severely hampering the functioning of various cancer services across the world. It is however, important to understand, cancer itself is a life-threatening condition, and there is a need to continue running cancer care services, at least for those who needed the most. Various clinical societies have put forward guidelines and protocols to help continue surgical services during the pandemic. The role of minimally invasive surgery (MIS) was initially questioned at the start of the pandemic, however gradually increasing evidence favored MIS as it reduced hospital stay and complication. Enhanced recovery programs which have been introduced to various fields of surgery to improve outcomes and reduce hospital stay. It plays an essential role in times like this, where the optimal usage of minimal resources is essential. We embraced these methods to ensure safety of our patients and staff and at the same time provide the highest standards of care. Here we are presenting our experience of running a colorectal surgical unit during these difficult times with emphasis on promotion of minimally invasive surgery, at the epicenter of the pandemic in India.
\end{abstract}

Keywords COVID $19 \cdot$ Minimally invasive surgery $\cdot$ Colorectal surgery $\cdot$ Outcomes

\section{Introduction}

The COVID-19 pandemic has impacted healthcare services around the world presenting with challenges to provide essential healthcare services. A recent survey of the WHO, which was conducted in 155 countries over a 3-week period in May, confirmed $42 \%$ of countries reporting partial or complete disruption of services for cancer treatment [1]. At the onset of the pandemic, various clinical societies across the world released guidelines to facilitate optimum delivery of healthcare in resource constrained settings taking cognizance of the risks faced by the healthcare providers. There is a need to incorporate and strictly enforce various strategies such as enhanced recovery programs which have demonstrated to lower

Avanish Saklani asaklani@hotmail.com

1 Division of Colorectal and Peritoneal Surface Oncology, Department of Surgical Oncology, Tata Memorial Centre, Homi Bhabha National Institute, Dr Ernest Borges Street, Parel, Mumbai 400012, India recovery time and post-operative complication rates, helping us to optimally utilize the minimum available resources without burdening the already strained health care infrastructure [3]. We present the outcomes of our patients who were treated in the Division of Colorectal and Peritoneal Surgery oncology services during this pandemic (Tables 1 and 2).

\section{Materials and Methods}

Tata Memorial Hospital is the apex oncology referral center of the country located in Mumbai, a city which has continued to share the maximum disease burden of COVID-19 of the nation till date. Prospectively maintained database of surgeries done in the department over a 60-day period between March and May 2020 was analyzed.

\section{Patient Selection}

All patients admitted and undergoing major elective as well as emergency surgeries under the Division of 
Table 1 Surgeries performed by the Division of Colorectal Surgery and peritoneal surface oncology over 2-month period

\begin{tabular}{|c|c|c|c|c|c|}
\hline \multirow{6}{*}{$\begin{array}{l}\text { Surgery } \\
\text { Surgical approach } \\
\text { - Minimally invasive surgery } \\
\text { - Open } \\
\text { Type of surgery }\end{array}$} & \multirow{2}{*}{\multicolumn{2}{|c|}{$\begin{array}{l}\text { Elective } \\
n=68\end{array}$}} & \multicolumn{2}{|c|}{ Emergency } & \multirow{2}{*}{$\begin{array}{l}\text { Total } \\
n=90\end{array}$} \\
\hline & & & \multicolumn{2}{|c|}{$\begin{array}{l}n=22 \\
9(40.9 \%)\end{array}$} & \\
\hline & \multicolumn{2}{|c|}{$43(63.2 \%)$} & $9(40$ & & $52(57.7 \%)$ \\
\hline & \multicolumn{2}{|c|}{$25(36.8 \%)$} & \multicolumn{2}{|c|}{$13(59.1 \%)$} & $38(42.3 \%)$ \\
\hline & \multicolumn{2}{|c|}{ Emergency } & \multicolumn{2}{|c|}{ Elective } & \\
\hline & Open & Lap & Open & Lap & \\
\hline - Staging laparoscopy and diversion stoma & 0 & 5 & 0 & 1 & 6 \\
\hline - Diversion stoma & 4 & 2 & 0 & 0 & 6 \\
\hline - Right hemicolectomy & 6 & 0 & 4 & 2 & 12 \\
\hline - Left hemicolectomy & 0 & 0 & $1^{\#}$ & 1 & 2 \\
\hline - Total colectomy & 1 & 0 & $4^{*}$ & 0 & 5 \\
\hline - Diagnostic laparoscopy & 0 & 1 & 0 & 3 & 4 \\
\hline - Exploratory laparotomy & 1 & 0 & 1 & 0 & 2 \\
\hline - Cytoreductive surgery & 0 & 0 & 1 & 0 & 1 \\
\hline - Appendectomy & 0 & 1 & 0 & 0 & 1 \\
\hline - Low anterior resection & 0 & 0 & 5 & 8 & 13 \\
\hline - Anterior resection & 0 & 0 & 4 & 10 & 14 \\
\hline - Intersphincteric resection & 0 & 0 & 0 & 4 & 4 \\
\hline - Abdominoperineal resection & 0 & 0 & 1 & 12 & 13 \\
\hline - Total pelvic exenteration & 0 & 0 & 1 & 0 & 1 \\
\hline - Hartmann's procedure & 0 & 0 & 0 & 2 & 2 \\
\hline - Stoma closure & 1 & 0 & 3 & 0 & 4 \\
\hline
\end{tabular}

* A simultaneous Whipple's procedure was done in one case

\# A simultaneous Left hepatectomy was done along with the case

Colorectal and Peritoneal Surface Oncology were included in the study. Exclusion criteria included patients with incomplete medical records, primarily admitted with another department for whom surgical cover was provided by the division of colorectal surgery and day care procedures.

\section{Primary Objective}

The primary objective of this study was to assess the outcome of the patients undergoing surgery during the study period. It included surgical duration, blood loss, post-operative hospital stay, and post-operative morbidity.

\section{Safety Protocols against COVID-19 Prevention}

Apart from the routine treatment and infection control protocols, emphasis was laid on the following in concert with the guidelines issued by the hospital infection control committee.

- After pre-anesthetic checkup, only patients with controlled comorbidities were scheduled for elective surgery after adequate optimization.

- Preoperative hospital visits and stay were minimized, once the surgical plan was made.

- In the initial 2 weeks of the study period, only symptomdriven testing for COVID-19 was in place at the hospital.
Table 2 Complications in patients who underwent surgery

\begin{tabular}{lll}
\hline Complication & Intervention & Clavien Dindo Grade \\
\hline Chyle leak & Pigtail insertion & Grade IIIa \\
Pelvic collection & Pigtail insertion & Grade IIIa \\
Peri-pancreatic collection & Pigtail insertion & Grade IIIa \\
Stoma prolapse & Re-exploration and refashioning of stoma & Grade IIIb \\
Anastomotic leak & Re-exploration & Grade IIIb \\
Anastomotic leak & Re-exploration & Grade IIIb \\
Anastomotic leak & Re-exploration & Grade IIIb \\
Small bowel injury & Re-exploration & Grade IIIb \\
Hemoperitoneum & Re-exploration & Grade IIIb \\
Perforation peritonitis & Exploratory laparotomy for perforation peritonitis & Grade V \\
(tumor perforation) & &
\end{tabular}


However, with the rapid surge of the pandemic, routine preoperative testing was started.

- Enhanced recovery after surgery (ERAS) were strictly followed. They included preoperative chest physiotherapy and anticoagulant prophylaxis against deep venous thrombosis.

- Preoperative carbohydrate loading, bowel preparation only when necessary, early enteral feeding in the postoperative period and early mobilization of the patients.

- Minimizing the entry of staff in the operating room.

- Ensuring the presence of only anesthetists during the time of intubation.

- Intubation using the preventive glass boxes at the head end.

- Ensuring adequate protective gears (N-95 masks, 3 ply masks, face shields) across all the staff in the operating room.

- Using HME (heat and moisture exchanger) filter with Airseal (Conmed Inc.US) during laparoscopic surgery to prevent exposure from surgical smoke.

- Minimizing the attendants with the patients during the hospital stay.

- Early discharge from hospital.

- Telephonic follow-up with the patients in the immediate post-operative period by a specialist nurse.

\section{Statistical Analysis}

The analysis was done using SPSS software (SPSS 22.0; SPSS Inc., Chicago, IL). Continuous variables are analyzed as means or medians and categorical variables are expressed as frequencies and percentages.

\section{Results}

A total of 90 patients underwent surgery over a 60-day period from March to May 2020. Out of the 90 surgeries performed, $68(75.6 \%)$ were elective surgeries and $22(24.4 \%)$ were emergency surgeries (Table 1). Majority of the surgeries were laparoscopic procedures accounting for 52 out of the 90 surgeries (57.8\%), while open surgeries accounted for the remaining 38 surgeries $(42.2 \%)$. Robotic surgeries and HIPEC were not performed during this period. Majority of the patients underwent a procedure with a curative intent ( 71 out of 90 , $78.8 \%$ ). Seventy percent of the cohort were males accounting for 63 out of the 90 patients. Most of the patients were ASA I $(56.7 \%)$, while $41.1 \%$ of the patients were ASA 2 with only 2 patients who were ASA 3 being operated in this time frame. Thirty-six of the 90 patients had a comorbidity such as diabetes mellitus, hypertension, asthma, and ischemic heart disease and were actively taking treatment for the same.
The median duration of surgery was 240 min $(60-$ $480 \mathrm{~min})$. The median blood loss was $225 \mathrm{ml}(50-2000 \mathrm{ml})$. The median post-operative stay was 5 days (1-30 days). The post-operative stay of the laparoscopic group and open surgery group were 4.3 day ( $1-15$ days) and 8.3 days (1-30 days) respectively. The median post-operative stay in the elective case and emergency cases were 5 days (1-30 days) and 6 days (1-21 days) respectively. Ten patients had a significant post op morbidity (Clavien Dindo Grade III and above) in the postoperative period (Tables 2). Four out of 43 rectal resections had a positive circumferential resection margin which corresponds to $9.3 \%$, which is slightly higher that our institutional rate of below $5 \%$, which probably is attributed to a selection bias of advanced cases during this period.

One of the patients who underwent exploratory laparotomy for perforation peritonitis died in the post-operative period. One patient was taken up for an emergency surgery without waiting for the result of COVID-19 (due to deterioration of symptoms) turned positive. Adequate precautions were taken in the preoperative and intraoperative period. No staff needed to be quarantined and his post-operative course was uneventful. No other patient developed the infection during their postoperative hospital stay.

\section{Discussion}

The COVID-19 pandemic has presented a unique set of challenges. India being a developing nation with limited healthcare infrastructure and over a billion-population responded by nationwide lockdown extending over 2 months putting severe socio-economic constraints.

The cancer care services had taken a hit after data emerged from China showing inferior outcomes in cancer patients undergoing treatment [4]. It is important to understand that cancer is also a life-threatening condition, which needs treatment with adequate precautionary measures and involvement of all oncological disciplines based on treatment protocols to obtain best possible outcomes [5].

Various guidelines have recommended that emergencies like intestinal occlusion, bowel perforation, peritonitis, massive GI bleeding, post-surgical complication, and postprocedural complications have to be attended with no delay. However, there have been contrasting views regarding the management of colorectal cancers. The European Society of Medical Oncology (ESMO) guidelines and American College of Surgeons (ACS) have advised to give priority to patient with cancers with the ACS suggesting surgeons to look at the Elective Surgery Acuity Scale (ESAS) from St. Louis University which include most cancers in Tier $3 \mathrm{a}$ and calls for non-post-ponement of surgery in these cases $[6,7]$. The NHS guidelines, on the other hand, on a more cautionary note has suggested surgery only for patients presenting in an 
emergency, colonic lesions, rectal cancers who are poor responders to NACTRT or 12-14 weeks post NACTRT [8].

The Tata Memorial Centre in India is the largest cancer center in the country and being the apex oncology referral center of the country; we had to continue to provide cancer care. However, our center did scale back our operations to function at $65 \%$ of routine capacity [9]. We also had to allocate beds for initial screening, testing, isolation, and treatment of COVID-19 patients.

The Division of Colorectal and Peritoneal Surface Oncology has a footfall of around 1500-1800 patients annually. During these difficult times, the resources allocated to the colorectal unit was cut down to a third. The department strives to provide the highest standards of cancer care while ensuring the safety of patients and healthcare providers. This resulted to adoption of best surgical practices suggested and put forward by various clinical societies and enforcing ERAS protocols. The adoption of MIS and enhanced recovery programs became mandatory for optimal usage of resources.

Tackling the reduction in hospital resources towards surgical care, the department managed to operate 90 patients over a 2 -month period. Emergency services were not hampered with $26.6 \%$ of the surgeries being done for emergent conditions like obstruction and bleeding. Guidelines from all the surgical societies have advocated the highest priority to emergency procedures [2]. The decision to perform a surgical procedure must be made in the context of numerous medical and logistic considerations taking into account the real risk of proceeding and the risk of delay. While COVID-19 testing was done for the semi-emergency surgeries, the urgent surgeries were never delayed for the lack of it proceeding with all the necessary precautions [10].

There has been hue and cry about the feasibility of minimally invasive surgery without clear guidelines for the same. We continued with MIS taking appropriate precautions, with $57.8 \%$ of the surgeries having been performed with laparoscopic approach. The proven benefits of MIS leading to reduced length of stay and complication should be strongly considered in these patients, in addition to the potential for ultrafiltration of the majority or all aerosolized particles. Filtration of aerosolized particles may be more difficult during open surgery [11].

The overall median post-operative stay was 5 days with laparoscopic group and open surgery group having a stay of 4.3 days (1-15 days) and 8.3 days (1-30 days) respectively. This was comparable with the average post-operative stay for colorectal surgeries. Various studies have demonstrated different lengths of stay in the hospital from 9 to 12 days [12] to 5 to 8 days [13]. The length of hospital stay also depends on whether the patient underwent emergency or elective surgery. Our series demonstrated the median hospital stay of 5.7 days (1-20 days) for elective surgeries and 8.5 days (2-30 days) for emergency surgeries which is comparable to published literature [14]. Strict enforcement of ERAS protocols especially during the postoperative period helped us achieve equivalent results. Javier et al. demonstrated that the implementation of ERAS protocol for colorectal surgery was associated with a significantly reduction of post-operative complications and length of stay [15]. The short-term outcomes of our cohort such as median blood loss, surgery duration, and post-operative morbidity were comparable with standard published literature [7].

We introduced a simplified enhanced recovery with early initiation of diet, mobilization, and discharge of patient's home with Jackson Pratt close suction drains. An interesting finding was even though the patients were keen on getting operated, they wanted to spend minimum time in the hospital and were not keen on a prolonged stay in the hospital after surgery. This made them receptive for an early discharge and care at home. A specialist nurse from our survivorship program called them on a regular basis to confirm their well-being.

At the same time, none of our patients barring one developed any COVID-19 infection in the perioperative period. We did not witness any increased morbidity or mortality due to COVID-19 infection. Even the safety of healthcare providers was optimum with no one testing positive for the same. After the initial 2 weeks, routine preoperative testing for COVID-19 was performed in concert with the SAGES guidelines which state that surgical patients should be tested pre-operatively for COVID-19 [11]. The patients who tested positive were kept quarantined at an associated medical facility to be operated after 2 weeks pending a negative result.

\section{Conclusion}

The challenges we face today as healthcare professionals are unparalleled, but we have successfully demonstrated the feasibility of cancer surgery during these difficult times by following strict protocols in testing, safety of personnel, and aggressive perioperative and post-operative care. The shortterm outcomes in our cohort are similar to those during non-COVID times and probably show us a way forward as treatment of cancer patients cannot be deferred for prolonged period of time.

Authors' Contributions All author equally contributed in preparation of this manuscript. Manuscript review was done by Dr. Avanish Saklani.

Data Availability Data available on request.

\section{Compliance with Ethical Standards}

Conflicts of Interest/Competing Interests None. 


\section{References}

1. Dyer O (2020) Covid-19: Pandemic is having "severe" impact on non- communicable disease care, WHO survey finds. BMJ 369: m2210. https://doi.org/10.1136/bmj.m2210

2. Geibel J (2020) COVID-19 guidance for surgeons

3. Pdeziwiatr M, Mavrikis J, Witowski J, Adamos A, Major P, Nowakowski M, Budzynski A (2018) Current status of enhanced recovery after surgery (ERAS) protocol in gastrointestinal surgery. Med Oncol 35(6):95

4. Zhang L, Zhu F, XIe L, SY X, Zhao JP, Zhou M Clinical characteristics of COVID-19-infected cancer patients: a retrospective case study in three hospitals within Wuhan, China. Ann Oncol

5. Desai S, Gupta A (2020) IASO COVID-19 guidelines (updated on $9^{\text {th }}$ April 2020) [published online ahead of print, 2020 may 6]. Indian J Surg Oncol:1-4. https://doi.org/10.1007/s13193-02001081-y

6. [Guideline] ESMO Management and treatment adapted recommendations in the Covid-19 era: colorectal Cancer (CRC). European Society of Medical Oncology Available at https://www.esmo.org/ guidelines/cancer-patient-management-during-the-covid-19pandemic/gastrointestinal-cancers-colorectal-cancer-crc-in-thecovid-19-era

7. [Guideline] COVID-19: guidance for triage of non - emergent surgical procedures. American College of Surgeons. Available at https://www.facs.org/covid-19/clinical-guidance/triage. March 17, 2020

8. [Guideline] Clinical guide to surgical prioritization during the coronavirus epidemic. Available at https://www.england.nhs.uk/ coronavirus/wp-content/uploads/sites/52/2020/03/C0221speciality-guide-surgical-prioritisation -v1.pdf. April 11, 2020
9. Pramesh CS, Badwe RA (2020) Cancer management in India during Covid-19. N Engl J Med:NEJMc2011595

10. Braga M, Vignali A, Zuliani W, Frasson M, DiSerio C (2005) Di Carlo V. laparoscopic versus open colorectal surgery: cost-benefit analysis in a single-center randomized trial. Ann Surg 242(6):890 896. https://doi.org/10.1097/01.sla.0000189573.23744.59

11. [Guideline] Pryor A. SAGES and EAES recommendations regarding surgical response to COVID-19 crisis. Society of American Gastrointestinal and Endoscopic Surgeons. Available at https:// www.sages.org/recommendations-surgical-response-covid-19. March 30, 2020

12. Burgdorf SK, Rosenberg J (2012) Short hospital stay after laparoscopic colorectal surgery without fast track. Minim Invasive Surg 2012:260273. https://doi.org/10.1155/2012/260273

13. Kelly M, Sharp L, Kelleher DF, Comber H (2012) Factors predicting hospital length-of-stay and readmission after colorectal resection : a population-based study of elective and emergency admissions. BMC Health Serv Res 12(77)

14. Ripolles-Melchor $J$ et al (2018) Enhanced recovery after surgery protocol versus conventional perioperative care in colorectal surgery a single center cohort study. Rev Bras Anestesiol 68(4):358 368

15. Braga M, Vignali A, Gianotti L et al (2002) Laparoscopic versus open colorectal surgery: a randomized trial on short-term outcome. Ann Surg 236(6):759-767. https://doi.org/10.1097/01.SLA. 0000036269.60340.AE

Publisher's Note Springer Nature remains neutral with regard to jurisdictional claims in published maps and institutional affiliations. 